\title{
Energy conservation for inhomogeneous Navier-Stokes equations
}

\author{
Jiaqi Yang ${ }^{1}$ \\ ${ }^{1}$ Northwestern Polytechnical University
}

May 5, 2020

\begin{abstract}
$\mathrm{n}$ this paper, we focus on the energy conservation for the weak solutions of inhomogeneous Navier-Stokes equations. It is proved that if the function of density belongs to $\$ \mathrm{~L}^{\wedge}\{\backslash \operatorname{infty}\}\left(0, \mathrm{~T} ; \mathrm{L}^{\wedge}\{\backslash \operatorname{infty}\}\left(\backslash \operatorname{mathbb}\{\mathrm{T}\}^{\wedge} \mathrm{N}\right)\right) \backslash \operatorname{cap} \mathrm{L}^{\wedge} \mathrm{p}\left(0, \mathrm{~T} ; \mathrm{W}^{\wedge}\{1, \mathrm{p}\}\left(\backslash \mathrm{mathbb}\{\mathrm{T}\}^{\wedge} \mathrm{N}\right)\right) \$$, and the function of velocity belongs to $\$ \mathrm{~L}^{\wedge} \mathrm{s}\left(0, \mathrm{~T} ; \mathrm{L} \wedge \mathrm{r}\left(\backslash\right.\right.$ mathbb $\left.\left.\{\mathrm{T}\}^{\wedge} \mathrm{N}\right)\right) \$$ with $\$ \backslash f 2 \mathrm{~s}+\backslash \mathrm{f} 2 \mathrm{r}=1 \$$, then the energy equality holds. This result can be seen as a inhomogeneous version for Shinbrot's criterion.
\end{abstract}

Hosted file

EE-INHOMO.pdf available at https://authorea.com/users/299670/articles/429154-energyconservation-for-inhomogeneous-navier-stokes-equations 